\title{
Clinical features of hepatocellular carcinoma associated with nonalcoholic fatty liver disease: a review of human studies
}

\author{
Kohei Oda $\cdot$ Hirofumi Uto $\cdot$ Seiichi Mawatari • \\ Akio Ido
}

Received: 28 November 2014/ Accepted: 17 December 2014/Published online: 10 January 2015

(C) Springer Japan 2015

\begin{abstract}
Most cases of hepatocellular carcinoma (HCC) in Japan develop in the background of chronic liver disease caused by hepatitis $\mathrm{C}$ virus (HCV). Recently, however, $\mathrm{HCV}$-associated $\mathrm{HCC}$ has been shown to be decreasing, while non-B and non-C HCC (NBNC-HCC), which is negative for $\mathrm{HCV}$ and hepatitis $\mathrm{B}$ virus infection, has increased. The main cause of NBNC-HCC is alcoholic liver disease, but the recent increase of NBNC-HCC is thought to be due to an increase in patients with nonalcoholic fatty liver disease (NAFLD). Approximately $10 \%$ of NAFLD cases involve nonalcoholic steatohepatitis (NASH), and NASH can progress to liver cirrhosis and its associated complications such as HCC. There are no accurate data on the percentage of NASH-related HCC among all-cause HCC in Japan, because no large-scale investigation has been performed. However, the rate is thought to be about $3 \%$ of overall HCC, which is lower than that in the United States. The incidence of $\mathrm{HCC}$ in patients with NASH-related cirrhosis is thought to be $2 \%$ per year, which is lower than that in HCV-related cirrhosis. Risks for NASH-related HCC include advanced hepatic fibrosis, older age, and being male. NAFLD that includes NASH is associated with metabolic syndrome, which includes obesity and diabetes, and metabolic syndrome is a risk factor for HCC. Genetic factors and dietary patterns may also be related to NASH-related HCC. Thus, regular HCC surveillance, as performed for patients with viral chronic liver disease, is required for patients with NAFLD,
\end{abstract}

K. Oda $\cdot$ H. Uto $(\varangle) \cdot$ S. Mawatari $\cdot$ A. Ido

Digestive and Lifestyle Diseases, Department of Human and Environmental Sciences, Kagoshima University Graduate School of Medical and Dental Sciences, 8-35-1 Sakuragaoka, Kagoshima, Kagoshima 890-8544, Japan

e-mail: hirouto@m2.kufm.kagoshima-u.ac.jp and diagnostic markers are required for simple, rapid and specific detection of NASH-related HCC.

Keywords Hepatocellular carcinoma - Nonalcoholic steatohepatitis · Nonalcoholic fatty liver disease

\section{Introduction}

Nonalcoholic fatty liver disease (NAFLD) has liver histology similar to that of alcoholic liver disease, even in patients with a history of no or a small amount of alcohol intake (30 g/day for males and $20 \mathrm{~g} /$ day for females when converted to ethanol) [1]. NAFLD is divided into two types: nonalcoholic fatty liver (NAFL), in which only fatty deposition occurs; and nonalcoholic steatohepatitis (NASH), in which hepatocellular ballooning and advanced hepatic fibrosis occur. NASH can also progress to advanced liver disease, including cirrhosis and hepatocellular carcinoma (HCC). Thus, NAFLD and NASH represent a wide-spectrum of disease that includes cirrhosis (NASH-related cirrhosis) and HCC (NASH-related HCC).

The prognosis in patients with NAFLD is mainly determined by liver disease-related death, including HCC, and cardiovascular disease; and the risks for complication with these diseases require evaluation in each patient to permit appropriate treatment [2-7]. Incidences of NAFLD have been reported worldwide at 6-35\% [8], and the incidence of NASH is estimated to be $3-5 \%$ of the general population $[8,9]$. NASH is currently the third most common indication for liver transplantation in the United States. However, the incidence of NAFLD is highest among chronic liver diseases in developed countries, and it has been predicted that NASH will be the most common underlying disease in patients requiring liver transplantation in 2020 [10]. In 
addition, the incidence of $\mathrm{HCC}$ is the third highest among malignant diseases [11-13], and an increase of NASHrelated HCC is an important concern.

The number of NAFLD patients has been increasing yearly, along with increased consumption of a Western diet and an increase in the number of obese patients. The incidence of NAFLD in Japan is estimated to be 20-30\% [14-17]. Approximately $10 \%$ of NAFLD cases are thought to be NASH and there are approximately two million patients with NASH in Japan [18]. Hepatitis C virus (HCV)-related chronic liver disease is currently the main cause of HCC in Japan, but a future increase of NASHrelated HCC is a concern [19]. In this review, we focus on the diagnosis, characteristics and risk factors of NASHrelated HCC, which will be increasingly important in the treatment of NAFLD.

\section{Incidence of HCC in patients with NAFLD/NASH}

NAFLD/NASH is a fatty liver disease that mainly develops due to obesity and insulin resistance. A focus has recently been placed on NASH-related HCC. HCC is one of the few cancers that are increasing in developed countries [20], and the incidence of HCC in males has risen threefold in the last 20 years in the United States [21]. This increase of HCC is partly due to the increased number of patients with NAFLD/NASH [22].

The number and percentage of patients with HCC induced by HCV has decreased, due to development of antiviral therapy and a decrease in new infection opportunities for hepatitis virus in Japan. In contrast, the number of cases of NBNC-HCC has increased [19], due to an increase in NASH-related HCC [23, 24]. In a study of 6,508 patients with NAFLD diagnosed by ultrasonography, the incidences of HCC were 0.19 and $0.51 \%$ at 8 and 12 years, respectively [25]. Arase et al. [26] found an incidence of NASH-related HCC of $0.63 \%$ over 8.2 years in 1,600 Japanese NAFLD patients aged 60 years old or older, and suggested that the high incidence was related to the older age of the subjects. The 5 -year incidence of HCC in patients with NASH-related cirrhosis has been reported to be $11.3 \%$, which is lower than that of $30.5 \%$ in patients with $\mathrm{HCV}$-related cirrhosis [27] (Fig. 1).

In other countries, Adams et al. [2] found that the incidence of HCC in patients with NAFLD was $0.5 \%$ over 7.6 years, and other reports also suggest an incidence of $<1 \%$ [4, 28-30]. Ekstedt et al. [4] found an incidence of HCC in patients with NASH of $2.8 \%$ over 13.7 years and the incidence of NASH-related HCC has been estimated to be $1-3 \%$ over 10 years $[29,30]$. In contrast, Ascha et al. [31] found a 3.2-year incidence of HCC of $12.8 \%$ in patients with NASH-related cirrhosis, and Sanyal et al. [32]

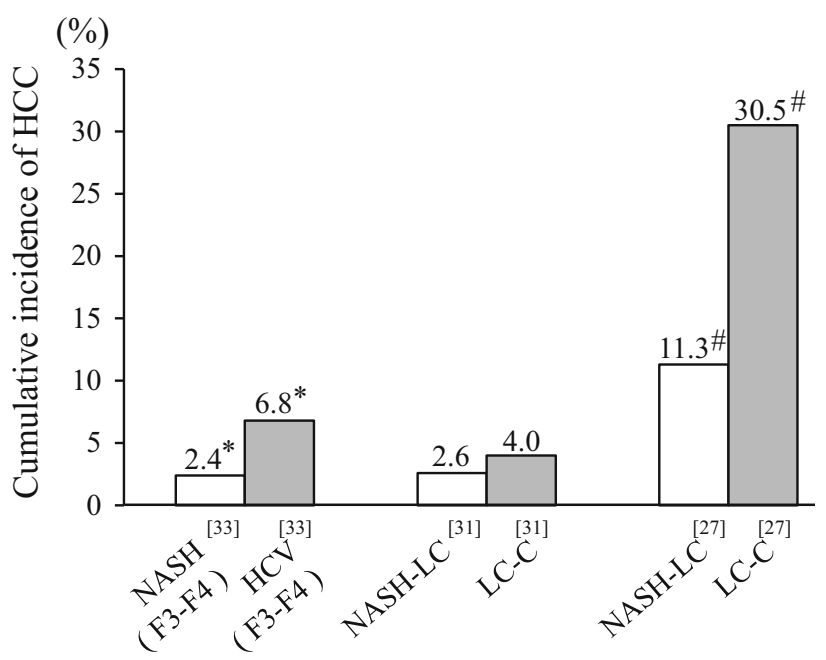

Fig. 1 Incidence of hepatocellular carcinoma in patients with nonalcoholic fatty liver disease or hepatitis $\mathrm{C}$ virus-related chronic liver disease. Asterisk 7.1-year cumulative incidence of HCC. Hash symbol 5-year cumulative incidence of HCC. HCC hepatocellular carcinoma, NASH nonalcoholic steatohepatitis, $\mathrm{HCV}$ hepatitis $\mathrm{C}$ virus-related chronic liver disease, $N A S H-L C$ nonalcoholic fatty liver disease-related liver cirrhosis, $L C-C$ hepatitis $\mathrm{C}$ virus-related liver cirrhosis, F3-F4 fibrosis stage 3-4 [reference number]

reported a 10-year incidence of $6.6 \%$. These results suggest that the incidence of NASH-related HCC is higher in patients in whom hepatic fibrosis has progressed to an advanced stage (Table 1).

The incidence of HCC in patients with NAFLD varies depending on patient background, but clearly increases when the disease is aggravated. HCC has a lower incidence in patients with NASH-related cirrhosis compared to those with HCV-related cirrhosis [31, 33, 34] (Fig. 1). However, HCC has been found to develop at a rate of approximately $2 \%$ per year in patients with NASH-related cirrhosis, and thus regular screening tests are required for patients with NASH because these patients are at high risk for $\mathrm{HCC}$.

\section{Risk factors for HCC in patients with NAFLD/NASH}

\section{Progression of hepatic fibrosis}

Progression of hepatic fibrosis is a major cause of liver disease-related death in patients with NASH [35], and is the most important risk factor for NASH-related HCC [34]. In a study of patients with NASH-related HCC, Hashimoto et al. [36] found advanced hepatic fibrosis in non-cancerous liver tissue in $88 \%$ of the subjects, and showed that advanced hepatic fibrosis was the most important risk factor for HCC [odds ratio (OR) 4.232]. Kawamura et al. [25] identified a platelet count of $\leq 150,000 / \mu \mathrm{L}$ as a risk factor for development of HCC in patients with NAFLD 
Table 1 Prevalence of HCC in patients with NAFLD or NASH

\begin{tabular}{|c|c|c|c|c|c|}
\hline References & $\begin{array}{l}\text { Type of } \\
\text { NAFLD }\end{array}$ & Number & Age of cohort & $\begin{array}{l}\text { HCC prevalence/years } \\
\text { of follow up }\end{array}$ & Comment \\
\hline \multicolumn{6}{|l|}{ Japan } \\
\hline Kawamura et al. [25] & NAFLD & 6508 & 49 (median) & $\begin{array}{l}0.02 \% / 4 \text { years, } 0.19 \% / \\
8 \text { years, } 0.51 \% / 12 \text { years } \\
(0.043 \% / 1 \text { year })\end{array}$ & Diagnosed by US \\
\hline Arase et al. [26] & NAFLD & 1600 & $62.5(9.5)^{\mathrm{a}}$ & $0.63 \% / 8.2$ years & Diagnosed by US \\
\hline Yatsuji et al. [27] & NASH-LC & 68 & $62.7(13.2)^{\mathrm{a}}$ & $11.3 \% / 5$ years & Biopsy-proven \\
\hline \multicolumn{6}{|c|}{ NAFLD/NASH patients outside Japan } \\
\hline Adams et al. [2] & NAFLD & 420 & $49(15)^{\mathrm{a}}$ & $0.48 \% / 7.6(4)^{\mathrm{a}}$ years & $\begin{array}{l}\text { Most cases were diagnosed } \\
\text { by imaging }\end{array}$ \\
\hline Ekstedt et al. [4] & NAFLD & 58 & $46.7(12.3)^{\mathrm{a}}$ & $0 \% / 13.7(1.3)^{\mathrm{a}}$ years & Biopsy-proven NAFLD \\
\hline Ong et al. [28] & NAFLD & 817 & $\begin{array}{r}\leq 40(50.4 \%), 40-\leq 60 \\
(34 \%),>60(15.6 \%)\end{array}$ & $0 \% / 8.4$ years & Analysis of cause of death \\
\hline Rafiq et al. [29] & NAFLD & $\begin{array}{l}173 \text { (NASH } \\
72)\end{array}$ & $50.2(14.5)^{\mathrm{a}}$ & $0.58 \% / 10.5$ years & $\begin{array}{l}\text { Biopsy-proven NAFLD, } \\
\text { death due to HCC }\end{array}$ \\
\hline Starley et al. [30] & NAFLD & - & - & $0.14 \%$ & Review (overall HCC prevalence) \\
\hline Ekstedt et al. [4] & NASH & $\begin{array}{l}71 \text { (NASH- } \\
\text { LC 4) }\end{array}$ & $54.5(12.4)^{\mathrm{a}}$ & $2.8 \% / 13.7(1.3)^{\mathrm{a}}$ years & Biopsy-proven NAFLD \\
\hline Starley et al. [30] & NASH & - & - & $1.6 \%$ & Review (overall HCC prevalence) \\
\hline Ascha et al. [31] & NASH-LC & 195 & 56.6 (median) & $\begin{array}{c}12.8 \% / 3.2 \text { years } \\
(2.6 \% / 1 \text { year })\end{array}$ & $\begin{array}{l}\text { Biopsy and/or radiological } \\
\text { evidence of cirrhosis }\end{array}$ \\
\hline Sanyal et al. [32] & NASH-LC & 152 & 55.3 (median) & $6.6 \% / 10$ years & Biopsy-proven \\
\hline Bhala et al. [33] & $\begin{array}{l}\text { NASH } \\
(\text { F3-F4) }\end{array}$ & 247 & $54.7(11.6)^{\mathrm{a}}$ & $2.4 \% / 7.1$ years & $\begin{array}{l}\text { Fibrosis stage } 3(47.7 \%) \\
\quad 4(52.2 \%)\end{array}$ \\
\hline
\end{tabular}

$\overline{H C C}$ hepatocellular carcinoma, NAFLD nonalcoholic fatty liver disease, NASH nonalcoholic steatohepatitis, LC liver cirrhosis, US ultrasonography

a Mean (SD)

[hazard ratio (HR) 7.19]. Thus, detection of advanced hepatic fibrosis in NASH cases is likely to be effective for early identification of patients at high risk for HCC.

In contrast, Sanyal et al. [37] found liver cirrhosis in non-cancerous liver tissue in $78 \%$ of patients who developed HCV-related HCC, but in only $46 \%$ of patients with NAFLD who developed HCC. Development of HCC in non-cirrhotic patients with NAFLD is also increasingly reported, and these patients have fatty changes and inflammatory findings in the portal region in the liver. Male patients with metabolic syndrome may also be at higher risk for HCC, even if they only have mild hepatic fibrosis of stage F0-F2 [22].

\section{Age and gender differences}

The prevalence of NASH is reported to be high in middleaged males and elderly females [38-40]. An age $\geq$ 60 years is a risk factor (HR 4.27) for development of HCC [25], and thus HCC should be considered in treatment of older patients with NASH.

The incidence of NASH is high in young males compared to young females (66 vs. $24 \%$ ), but the incidence is higher in females $\geq 50$ years of age compared to males $\geq 50$ years of ages (76 vs. $34 \%$ ) [40]. This is thought to be because many females develop NASH due to rapid progression of hepatic fibrosis after menopause. However, despite the incidence of NASH-related cirrhosis being higher in females, the incidence of concomitant HCC is higher in males (62 vs. $38 \%$ ) [36], which suggests that males have a high risk of NASH-related HCC. Further, even young males with mild progression of hepatic fibrosis may be at higher risk of development of HCC compared to females [41]. Thus, male NAFLD patients with other risk factors should be especially carefully monitored for onset of HCC [42].

\section{Obesity and metabolic syndrome}

Obesity is a strong risk factor for HCC [43]. A cohort study of 900,000 people living in North America showed high mortality in patients with HCC with BMI $>35 \mathrm{~kg} / \mathrm{m}^{2}$ (HR 4.52 in males and 1.68 in females) [44]. In another cohort study with 578,700 subjects in Northern Europe, 266 subjects developed HCC during the 12-year observation period, and a high BMI [relative risk (RR) 1.39] and metabolic 
syndrome (RR 1.35) were confirmed as risk factors for HCC [45]. In a multicenter collaborative study in Europe with 359,525 subjects, a weight increase in adulthood was found to be a risk factor for HCC (RR 2.48) [46]. Metaanalyses in the United States, Europe and Asia have also suggested that an overweight status is a risk factor for HCC (OR 1.89) [47]. In a study of 3,649 HCC patients and 743 patients with intrahepatic cholangiocarcinoma in the United States, metabolic syndrome was found to be a risk factor for development of HCC (OR 2.13) and intrahepatic cholangiocarcinoma (OR 1.56) [48]. These findings clearly suggest that obesity and metabolic syndrome are closely related to $\mathrm{HCC}$.

In a review article, Starley et al. [30] found that $34-54 \%, 18-29 \%, 27-37 \%$, and $9 \%$ of NASH patients showed no progression, improvement, aggravation of hepatic fibrosis, and development of liver cirrhosis, respectively, over 5.6 years. These authors also suggested that obesity and high BMI are independent risk factors for progression of hepatic fibrosis [30]. A study of NAFLD/ NASH patients who underwent liver biopsy several times also showed that decreases in BMI and waist size were independent factors associated with no progression of hepatic fibrosis [49], and a $7 \%$ weight decrease improves the NAFLD activity score [50]. Thus, weight control is important in treatment of NAFLD/NASH, and hepatic fibrosis progression and the high incidence of HCC may be ameliorated by decreasing weight in NAFLD/NASH patients.

\section{Diabetes}

In a large-scale cohort study, the risk of malignant disease was found to be higher in patients with diabetes compared to those without diabetes (1.27 times in males and 1.21 times in females). Diabetes also led to a higher risk of HCC in males and females (HRs of 2.24 and 1.94, respectively), and was more strongly associated with development of HCC among malignant tumors [51]. A meta-analysis also suggested that diabetes increases the risk of HCC (RR 2.38) [52] and diabetes is a risk factor for HCC in patients with NAFLD (HR 3.21) [25].

In studies outside Japan, diabetes has also been shown to be an independent risk factor for HCC [53, 54]. A study in the United States found that 74 and $22.2 \%$ of type II diabetes patients had NAFLD and NASH, respectively [9], and patients with a complication of diabetes are at high risk for NAFLD (HR 1.98) and HCC (HR 2.16) [55]. A metaanalysis also showed that many patients with diabetes develop HCC concomitantly (HR 2.01) [56].

These findings show that onset of HCC should be monitored in NAFLD/NASH patients, and especially in those with diabetes. The incidence of future complication of diabetes is also high in NAFLD [14], and diabetes should be viewed as an important complication in the treatment of NAFLD/NASH.

\section{Hyperlipidemia}

Excess intake of cholesterol [57] and saturated fatty acids [58] are both risk factors for NAFLD, and total and lowdensity lipoprotein (LDL) cholesterol may be increased in patients with NAFLD [59]. Fatty liver is also an independent risk factor for an increase in small dense LDL [60]. Thus, hyperlipidemia is associated with NAFLD [17], but there is no direct evidence from human studies that shows that hyperlipidemia contributes to onset of HCC.

\section{Genetic factors}

Genome-wide association studies (GWAS) have suggested that patatin-like phospholipase domain-containing protein 3 (PNPLA3) is a genetic factor that contributes to the development and progression of NASH [61-63]. PNPLA3 is present on chromosome 22 and is involved in metabolism of triglyceride. A single nucleotide polymorphism (SNP) (rs738409) of PNPLA3 is closely related to fatty liver, and the fat level in the liver increases by approximately $100 \%$ in patients who are homozygous for this risk allele of PNPLA3. This SNP is also involved in progression of fibrosis in NAFLD [64, 65], and is significantly correlated with NAFLD of Matteoni classification type 4 [63]. The $\mathrm{C}<\mathrm{G}$ variation in SNP rs738409 of PNPLA3 also increases the risk of HCC in patients with NAFLD [66]. Compared to the CC allele, the GC and GG alleles have risks for HCC with ORs of 2.52 and 12.19, respectively. Thus, SNP rs738409 of PNPLA3 appears to be related to development of HCC in patients with NAFLD/NASH.

\section{Alcohol intake and smoking}

Alcohol intake may play a protective role against fatty liver [67], but persistent alcohol abuse is a well-established etiologic factor in HCC. A prospective study of NASHrelated cirrhosis also showed that the risk of HCC in subjects with a small amount of alcohol intake (occasional drinking) was higher (HR 3.6) than that for subjects who did not drink alcohol [31]. Furthermore, the incidence of advanced hepatic fibrosis is high in smokers [68], and the percentage of patients with a smoking history has been found to be higher among those with newly developed NAFLD compared to those without NAFLD over a 10-year study period [15]. Tobacco smoking is a significant risk factor for development of HCC, and significant dosedependent and duration-dependent relationships between 
tobacco use and HCC risk have been found [69]. After exclusion of subjects who were daily drinkers and were positive for hepatitis B virus (HBV) or $\mathrm{HCV}$ infection, the risk of $\mathrm{HCC}$ associated with long-term smoking was 1.85fold higher than that in subjects who had never smoked (HR $=1.85,95 \%$ CI 0.66-5.18) [69]. Thus, alcohol intake and smoking may promote development of NASHrelated HCC.

\section{Inflammatory cytokines}

The "two hit" theory [70] and the "multiple parallel hits" hypothesis [71] have been proposed for the pathogenesis of NASH. The multiple parallel hits hypothesis suggests that multiple equilibrium hits from the intestinal tract and fat tissues are involved in progression of $\mathrm{NASH}$, and that changes such as increased inflammatory cytokines (TNF $\alpha$, IL-6 and resistin) and decreased adiponectin, an antiinflammatory cytokine, may promote hepatocarcinogenesis [72-76]. Changes in intestinal bacteria associated with obesity might also promote development of HCC [77], although further studies in humans are needed.

\section{Fructose}

The increased intake of fructose in recent years is related to weight increases and development of obesity in childhood and adolescence [78-80]. Excessive intake of fructose may aggravate NAFLD [81-84]. Patients with histologically diagnosed NAFLD have increased progression of hepatic fibrosis if their fructose level is high, despite having a mild level of fatty liver. Excessive fructose intake also appears to aggravate the clinical conditions of NAFLD/NASH [85]. These findings suggest that excess fructose may contribute to development of NAFLD and progression of hepatic fibrosis, and may be related to onset of HCC.

\section{Iron metabolism}

In patients with NAFLD, the ferritin level is closely correlated with the degree of hepatic fibrosis [86-88]. Iron deposition in liver tissues is higher in NASH than in NAFL, and excess iron produces reactive oxygen species and aggravates hepatic disorder through oxidative stress. The iron level in liver tissues in NASH patients with HCC is also significantly higher than that in NASH patients without HCC [89]. Excess iron is thought to be a risk for progression of hepatic fibrosis and hepatocarcinogenesis in $\mathrm{NASH}$, and thus patients with a high serum ferritin level, which suggests excess iron, should be screened carefully for the development of HCC.
Characteristics and diagnosis of HCC in patients with NAFLD/NASH

Appropriate selection of patients with advanced hepatic fibrosis is important in screening for NAFLD/NASH-related HCC. Aspartate aminotransferase (AST), alanine aminotransferase (ALT) and $\gamma$-glutamyl transpeptidase $(\gamma$ GTP) levels do not reflect the severity of NAFLD, and such liver function test results are occasionally within normal limits in patients with NAFLD/NASH [90, 91]. Therefore, longitudinal evaluation with tumor markers and hepatic fibrosis markers is important for identification of patients at high risk for NASH-related HCC.

\section{Tumor markers}

Of 19 NASH patients who developed HCC during an observation period, abnormal levels of $\alpha$-fetoprotein (AFP, $>10 \mathrm{ng} / \mathrm{mL}$ ) and protein induced by Vitamin $\mathrm{K}$ absence or antagonists (PIVKA)-II [also known as des- $\gamma$-carboxy prothrombin (DCP), $>40 \mathrm{mAU} / \mathrm{mL}$ ] were found in ten $(53 \%)$ and nine $(47 \%)$, respectively, upon diagnosis of $\mathrm{HCC}$, suggesting that the positive rates for these tumor markers are not particularly high in patients with NASHrelated HCC [92]. Also, the increase in average AFP from before to after development of HCC $(6.0-10.5 \mathrm{ng} / \mathrm{mL})$ was not significant, but the increase of PIVKA-II (18-34 mAU/ $\mathrm{mL})$ was significant. These findings suggest that an increase in PIVKA-II may be useful to detect NASHrelated HCC, but that screening for NASH-related HCC using only tumor markers is insufficient [92].

\section{Platelet count}

Platelet count is a useful index for stage of hepatic fibrosis in NAFLD. Compared to NAFL patients, NASH patients have lower platelet counts, and progression of NASH should be suspected in patients with a decrease in platelet count over time. However, this requires careful confirmation, because the decrease in platelet count is minor in many NAFLD/NASH patients compared to patients with viral hepatitis, such as chronic hepatitis $\mathrm{C}$. A platelet count of $150,000 / \mu \mathrm{L}$ suggests hepatic fibrosis of stage $\mathrm{F} 2$ in patients with chronic hepatitis $\mathrm{C}$, whereas platelet counts of $192,000 / \mu \mathrm{L}$ and $153,000 / \mu \mathrm{L}$ suggest hepatic fibrosis of stages F3 (sensitivity $62.7 \%$, specificity $76.3 \%$ ) and F4 (sensitivity $80.5 \%$, specificity $88.8 \%$ ), respectively, in NAFLD patients [93]. A platelet count of $\leq 150,000 / \mu \mathrm{L}$ may be a risk factor for the development of HCC (HR 7.19) [25]. NAFLD/NASH patients with a platelet count of $\leq 150,000 / \mu \mathrm{L}$ may have liver cirrhosis and may be at high risk for HCC. 


\section{Blood biomarkers}

Serum concentrations of cytokeratin 18 fragment [94], adiponectin [95], high sensitivity CRP [96], thioredoxin [97], and MnSOD [98] are useful for diagnosis of NASH. In a large-scale study, cytokeratin 18 fragment was shown to a useful single diagnostic marker for NASH [99]. A glycosylation marker has also been suggested to be effective for evaluating the progression of hepatic fibrosis and the disease stages of viral hepatitis and NAFLD [100, 101]. Further accumulation of evidence is required to determine which marker is most useful for screening of NASH-related HCC.

\section{Hepatic fibrosis scoring systems}

Scoring systems using multiple factors are useful for determination of the disease stage of NAFLD/NASH. The NAFLD fibrosis score (NFS) [102], FIB-4 index [103, 104], and NAFIC score [87] increase with progression of NASH, and the NFS and FIB-4 index are useful as prognostic markers [105]. Kawamura et al. [25] found an incidence of HCC in patients with NAFLD of $0.043 \%$ / year, and showed that the incidence of HCC in patients diagnosed with advanced hepatic fibrosis based on an ASTto-platelet ratio index (APRI) of $>1.5$ was significantly higher than that in patients without advanced hepatic fibrosis (APRI $\leq 1.5$ ). Using the FIB-4 index, Yoneda et al. [106] found advanced hepatic fibrosis (F3-F4) in $16.1 \%$ of NAFLD patients, even when ALT levels were normal. These findings suggest that these scoring systems are useful for prediction of hepatic fibrosis and HCC.

\section{Imaging tests}

Transient elastography is useful for noninvasive evaluation of hepatic fibrosis based on measurement of the stiffness of the liver by ultrasonography [107, 108]. NASH can be diagnosed by detection of dysfunction of Kupffer cells using contrast-enhanced ultrasonography [109]. Magnetic resonance imaging (MRI) is also useful for evaluation of progression of hepatic fibrosis in NAFLD [110]. Since it is difficult to perform liver biopsy for all NAFLD patients, scoring systems and imaging tests that enable estimation of the degree of hepatic fibrosis are useful to identify patients at high risk for HCC.

\section{Conclusion}

The increase in the number of NAFLD/NASH patients suggests that these liver diseases will be more important in the future. The risk of HCC in NAFLD/NASH patients is lower than that in patients with HCV-related chronic liver disease, but the number of patients with NAFLD/NASH is large, and thus the percentage of patients with NASHrelated HCC among all HCC patients is likely to increase. However, there are no diagnostic markers for simple, rapid and specific detection of NAFLD/NASH-related HCC. Furthermore, the frequency of HCC surveillance for NAFLD patients is low compared to patients with HCV-related chronic liver disease and alcoholic liver disease [111]. This emphasizes the importance of development of accurate blood tests and imaging tests for HCC surveillance in NAFLD/NASH patients.

Conflict of interest Kohei Oda, Hirofumi Uto, Seiichi Mawatari and Akio Ido declare that they have no conflict of interest.

\section{References}

1. Sanyal AJ, Brunt EM, Kleiner DE, et al. Endpoints and clinical trial design for nonalcoholic steatohepatitis. Hepatology. 2011;54:344-53.

2. Adams LA, Lymp JF, StSauver J, et al. The natural history of nonalcoholic fatty liver disease: a population-based cohort study. Gastroenterology. 2005;129:113-21.

3. Targher G, Bertolini L, Poli F, et al. Nonalcoholic fatty liver disease and risk of future cardiovascular events among type 2 diabetic patients. Diabetes. 2005;54:3541-6.

4. Ekstedt M, Franzén LE, Mathiesen UL, et al. Long-term followup of patients with NAFLD and elevated liver enzymes. Hepatology. 2006;44:865-73.

5. Argo CK, Argo CK, Northup PG, et al. Systematic review of risk factors for fibrosis progression in non-alcoholic steatohepatitis. J Hepatol. 2009;51:371-9.

6. Söderberg C, Stål P, Askling J, et al. Decreased survival of subjects with elevated liver function tests during a 28 -year follow-up. Hepatology. 2010;51:595-602.

7. Musso G, Gambino R, Cassader M, et al. Meta-analysis: natural history of non-alcoholic fatty liver disease (NAFLD) and diagnostic accuracy of non-invasive tests for liver disease severity. Ann Med. 2011;43:617-49.

8. Vernon G, Baranova A, Younossi ZM. Systematic review: the epidemiology and natural history of non-alcoholic fatty liver disease and non-alcoholic steatohepatitis in adults. Aliment Pharmacol Ther. 2011;34:274-85.

9. Williams CD, Stengel J, Asike MI, et al. Prevalence of nonalcoholic fatty liver disease and nonalcoholic steatohepatitis among a largely middle-aged population utilizing ultrasound and liver biopsy: a prospective study. Gastroenterology. 2011;140:124-31.

10. Charlton MR, Burns JM, Pedersen RA, et al. Frequency and outcomes of liver transplantation for nonalcoholic steatohepatitis in the United States. Gastroenterology. 2011;141:1249-53.

11. Bosch FX, Ribes J, Díaz M, et al. Primary liver cancer: worldwide incidence and trends. Gastroenterology. 2004;127:S5-16.

12. Jemal A, Bray F, Center MM, et al. Global cancer statistics. CA Cancer J Clin. 2011;61:69-90.

13. Karagozian R, Derdák Z, Baffy G. Obesity-associated mechanisms of hepatocarcinogenesis. Metabolism. 2014;63:607-17.

14. Imamura $\mathrm{Y}$, Uto $\mathrm{H}$, Hiramine $\mathrm{Y}$, et al. Increasing prevalence of diabetes mellitus in association with fatty liver in a Japanese population. J Gastroenterol. 2014;49:1406-13. 
15. Hamabe A, Uto H, Imamura $\mathrm{Y}$, et al. Impact of cigarette smoking on onset of nonalcoholic fatty liver disease over a 10-year period. J Gastroenterol. 2011;46:769-78.

16. Eguchi Y, Hyogo H, Ono M, et al. Prevalence and associated metabolic factors of nonalcoholic fatty liver disease in the general population from 2009 to 2010 in Japan: a multicenter large retrospective study. J Gastroenterol. 2012;47:586-95.

17. Hamaguchi M, Takeda N, Kojima T, et al. Identification of individuals with non-alcoholic fatty liver disease by the diagnostic criteria for the metabolic syndrome. World J Gastroenterol. 2012;18:1508-16.

18. Okanoue T, Umemura A, Yasui K, et al. Nonalcoholic fatty liver disease and nonalcoholic steatohepatitis in Japan. J Gastroenterol Hepatol. 2011;26:153-62.

19. Tateishi R, Okanoue T, Fujiwara N, et al. Clinical characteristics, treatment, and prognosis of non-B, non-C hepatocellular carcinoma: a large retrospective multicenter cohort study. J Gastroenterol. 2014 (Epub ahead of print).

20. Zhang ZJ, Zheng ZJ, Shi R, et al. Metformin for liver cancer prevention in patients with type 2 diabetes: a systematic review and meta-analysis. J Clin Endocrinol Metab. 2012;97:2347-53.

21. Altekruse SF, McGlynn KA, Reichman ME. Hepatocellular carcinoma incidence, mortality, and survival trends in the United States from 1975 to 2005. J Clin Oncol. 2009;27:1485-91.

22. Baffy G, Brunt EM, Caldwell SH. Hepatocellular carcinoma in non-alcoholic fatty liver disease: an emerging menace. J Hepatol. 2012;56:1384-91.

23. Hashimoto E, Tokushige K. Hepatocellular carcinoma in nonalcoholic steatohepatitis: growing evidence of an epidemic? Hepatol Res. 2012;42:1-14.

24. Nagaoki Y, Hyogo H, Aikata H, et al. Recent trend of clinical features in patients with hepatocellular carcinoma. Hepatol Res. 2012;42:368-75.

25. Kawamura Y, Arase Y, Ikeda K, et al. Large-scale long-term follow-up study of Japanese patients with non-alcoholic Fatty liver disease for the onset of hepatocellular carcinoma. Am J Gastroenterol. 2012;107:253-61.

26. Arase Y, Kobayashi M, Suzuki F, et al. Difference in malignancies of chronic liver disease due to non-alcoholic fatty liver disease or hepatitis C in Japanese elderly patients. Hepatol Res. 2012;42:264-72.

27. Yatsuji S, Hashimoto E, Tobari M, et al. Clinical features and outcomes of cirrhosis due to non-alcoholic steatohepatitis compared with cirrhosis caused by chronic hepatitis C. J Gastroenterol Hepatol. 2009;24:248-54.

28. Ong JP, Pitts A, Younossi ZM. Increased overall mortality and liver-related mortality in non-alcoholic fatty liver disease. J Hepatol. 2008;49:608-12.

29. Rafiq N, Bai C, Fang Y, et al. Long-term follow-up of patients with nonalcoholic fatty liver. Clin Gastroenterol Hepatol. 2009;7:234-8.

30. Starley BQ, Calcagno CJ, Harrison SA. Nonalcoholic fatty liver disease and hepatocellular carcinoma: a weighty connection. Hepatology. 2010;51:1820-32.

31. Ascha MS, Hanouneh IA, Lopez R, et al. The incidence and risk factors of hepatocellular carcinoma in patients with nonalcoholic steatohepatitis. Hepatology. 2010;51:1972-8.

32. Sanyal AJ, Banas C, Sargeant C, et al. Similarities and differences in outcomes of cirrhosis due to nonalcoholic steatohepatitis and hepatitis C. Hepatology. 2006;43:682-9.

33. Bhala N, Angulo P, van der Poorten D, et al. The natural history of nonalcoholic fatty liver disease with advanced fibrosis or cirrhosis: an international collaborative study. Hepatology. 2011;54:1208-16.

34. White DL, Kanwal F, El-Serag HB. Association between nonalcoholic fatty liver disease and risk for hepatocellular cancer, based on systematic review. Clin Gastroenterol Hepatol. 2012;10:1342-59.

35. Younossi ZM, Stepanova M, Rafiq N, et al. Pathologic criteria for nonalcoholic steatohepatitis: interprotocol agreement and ability to predict liver-related mortality. Hepatology. 2011;53: 1874-82.

36. Hashimoto E, Yatsuji S, Tobari M, et al. Hepatocellular carcinoma in patients with nonalcoholic steatohepatitis. J Gastroenterol. 2009;44:89-95.

37. Sanyal A, Poklepovic A, Moyneur E, et al. Population-based risk factors and resource utilization for HCC: US perspective. Curr Med Res Opin. 2010;26:2183-91.

38. Jimba S, Nakagami T, Takahashi M, et al. Prevalence of nonalcoholic fatty liver disease and its association with impaired glucose metabolism in Japanese adults. Diabet Med. 2005;22:1141-5.

39. Yatsuji S, Hashimoto E, Tobari M, et al. Influence of age and gender in Japanese patients with non-alcoholic steatohepatitis. Hepatol Res. 2007;37:1034-43.

40. Hashimoto E, Tokushige K. Prevalence, gender, ethnic variations, and prognosis of NASH. J Gastroenterol. 2011;46:63-9.

41. Yasui K, Hashimoto E, Komorizono Y, et al. Characteristics of patients with nonalcoholic steatohepatitis who develop hepatocellular carcinoma. Clin Gastroenterol Hepatol. 2011;9:428-33.

42. Duan XY, Qiao L, Fan JG. Clinical features of nonalcoholic fatty liver disease-associated hepatocellular carcinoma. Hepatobiliary Pancreat Dis Int. 2012;11:18-27.

43. Turati F, Talamini R, Pelucchi C, et al. Metabolic syndrome and hepatocellular carcinoma risk. Br J Cancer. 2013;108:222-8.

44. Calle EE, Rodriguez C, Walker-Thurmond K, et al. Overweight, obesity, and mortality from cancer in a prospectively studied cohort of U.S. adults. N Engl J Med. 2003;348:1625-38.

45. Borena W, Strohmaier S, Lukanova A, et al. Metabolic risk factors and primary liver cancer in a prospective study of 578,700 adults. Int J Cancer. 2012;131:193-200.

46. Schlesinger S, Aleksandrova K, Pischon T, et al. Abdominal obesity, weight gain during adulthood and risk of liver and biliary tract cancer in a European cohort. Int $\mathrm{J}$ Cancer. 2013;132:645-57.

47. Larsson SC, Wolk A. Overweight, obesity and risk of liver cancer: a meta-analysis of cohort studies. Br J Cancer. 2007;97:1005-8.

48. Welzel TM, Graubard BI, Zeuzem S, et al. Metabolic syndrome increases the risk of primary liver cancer in the United States: a study in the SEER-Medicare database. Hepatology. 2011;54: 463-71.

49. Wong VW, Wong GL, Choi PC, et al. Disease progression of non-alcoholic fatty liver disease: a prospective study with paired liver biopsies at 3 years. Gut. 2010;59:969-74.

50. Promrat K, Kleiner DE, Niemeier HM, et al. Randomized controlled trial testing the effects of weight loss on nonalcoholic steatohepatitis. Hepatology. 2010;51:121-9.

51. Inoue M, Iwasaki M, Otani T, et al. Diabetes mellitus and the risk of cancer: results from a large-scale population-based cohort study in Japan. Arch Intern Med. 2006;166:1871-7.

52. Noto H, Osame K, Sasazuki T, et al. Substantially increased risk of cancer in patients with diabetes mellitus: a systematic review and meta-analysis of epidemiologic evidence in Japan. J Diabetes Complicat. 2010;24:345-53.

53. Chen HF, Chen P, Li CY. Risk of malignant neoplasms of liver and biliary tract in diabetic patients with different age and sex stratifications. Hepatology. 2010;52:155-63.

54. Koh WP, Wang R, Jin A, et al. Diabetes mellitus and risk of hepatocellular carcinoma: findings from the Singapore Chinese Health Study. Br J Cancer. 2013;108:1182-8.

55. El-Serag HB, Tran T, Everhart JE. Diabetes increases the risk of chronic liver disease and hepatocellular carcinoma. Gastroenterology. 2004;126:460-8. 
56. Wang C, Wang X, Gong G, et al. Increased risk of hepatocellular carcinoma in patients with diabetes mellitus: a systematic review and meta-analysis of cohort studies. Int $\mathrm{J}$ Cancer. 2012;130:1639-48.

57. Yasutake K, Nakamuta M, Shima Y, et al. Nutritional investigation of non-obese patients with non-alcoholic fatty liver disease: the significance of dietary cholesterol. Scand J Gastroenterol. 2009;44:471-7.

58. Leamy AK, Egnatchik RA, Young JD. Molecular mechanisms and the role of saturated fatty acids in the progression of nonalcoholic fatty liver disease. Prog Lipid Res. 2013;52:165-74.

59. Enjoji M, Nakamuta M. Is the control of dietary cholesterol intake sufficiently effective to ameliorate nonalcoholic fatty liver disease? World J Gastroenterol. 2010;16:800-3.

60. Hosoyamada K, Uto H, Imamura Y, et al. Fatty liver in men is associated with high serum levels of small, dense low-density lipoprotein cholesterol. Diabetol Metab Syndr. 2012;4:34.

61. Romeo S, Kozlitina J, Xing C, et al. Genetic variation in PNPLA3 confers susceptibility to nonalcoholic fatty liver disease. Nat Genet. 2008;40:1461-5.

62. Hotta K, Yoneda M, Hyogo H, et al. Association of the rs738409 polymorphism in PNPLA3 with liver damage and the development of nonalcoholic fatty liver disease. BMC Med Genet. 2010;11:172.

63. Kawaguchi T, Sumida Y, Umemura A, et al. Genetic polymorphisms of the human PNPLA3 gene are strongly associated with severity of non-alcoholic fatty liver disease in Japanese. PLoS ONE. 2012;7:e38322.

64. Sookoian S, Pirola CJ. Meta-analysis of the influence of I148M variant of patatin-like phospholipase domain containing 3 gene (PNPLA3) on the susceptibility and histological severity of nonalcoholic fatty liver disease. Hepatology. 2011;53:1883-94.

65. Kitamoto T, Kitamoto A, Yoneda M, et al. Genome-wide scan revealed that polymorphisms in the PNPLA3, SAMM50, and PARVB genes are associated with development and progression of nonalcoholic fatty liver disease in Japan. Hum Genet. 2013;132:783-92.

66. Liu YL, Patman GL, Leathart JB, et al. Carriage of the PNPLA3 rs $738409 \mathrm{C}>\mathrm{G}$ polymorphism confers an increased risk of nonalcoholic fatty liver disease associated hepatocellular carcinoma. J Hepatol. 2014;61:75-81.

67. Hiramine Y, Imamura Y, Uto H, et al. Alcohol drinking patterns and the risk of fatty liver in Japanese men. J Gastroenterol. 2011;46:519-28.

68. Zein CO, Unalp A, Colvin R, et al. Smoking and severity of hepatic fibrosis in nonalcoholic fatty liver disease. J Hepatol. 2011;54:753-9.

69. Koh WP, Robien K, Wang R, et al. Smoking as an independent risk factor for hepatocellular carcinoma: the Singapore Chinese Health Study. Br J Cancer. 2011;105:1430-5.

70. Day CP, James OF. Steatohepatitis: a tale of two "hits"? Gastroenterology. 1998;114:842-5.

71. Tilg H, Moschen AR. Evolution of inflammation in nonalcoholic fatty liver disease: the multiple parallel hits hypothesis. Hepatology. 2010;52:1836-46.

72. Harrison SA. Liver disease in patients with diabetes mellitus. J Clin Gastroenterol. 2006;40:68-76.

73. Bugianesi E. Non-alcoholic steatohepatitis and cancer. Clin Liver Dis. 2007;11:191-207.

74. Vázquez-Vela ME, Torres N, Tovar AR. White adipose tissue as endocrine organ and its role in obesity. Arch Med Res. 2008;39:715-28.

75. Kamada Y, Takehara T, Hayashi N. Adipocytokines and liver disease. J Gastroenterol. 2008;43:811-22.
76. Park EJ, Lee JH, Yu GY, et al. Dietary and genetic obesity promote liver inflammation and tumorigenesis by enhancing IL6 and TNF expression. Cell. 2010;140:197-208.

77. Yoshimoto S, Loo TM, Atarashi K, et al. Obesity-induced gut microbial metabolite promotes liver cancer through senescence secretome. Nature. 2013;499:97-101.

78. Elliott SS, Keim NL, Stern JS, et al. Fructose, weight gain, and the insulin resistance syndrome. Am J Clin Nutr. 2002;76:911-22.

79. Bray GA, Nielsen SJ, Popkin BM. Consumption of high-fructose corn syrup in beverages may play a role in the epidemic of obesity. Am J Clin Nutr. 2004;79:537-43.

80. Harrington S. The role of sugar-sweetened beverage consumption in adolescent obesity: a review of the literature. J Sch Nurs. 2008;24:3-12.

81. Ouyang X, Cirillo P, Sautin Y, et al. Fructose consumption as a risk factor for non-alcoholic fatty liver disease. J Hepatol. 2008;48:993-9.

82. Thuy S, Ladurner R, Volynets V, et al. Nonalcoholic fatty liver disease in humans is associated with increased plasma endotoxin and plasminogen activator inhibitor 1 concentrations and with fructose intake. J Nutr. 2008;138:1452-5.

83. Vos MB, Lavine JE. Dietary fructose in nonalcoholic fatty liver disease. Hepatology. 2013;57:2525-31.

84. Ishimoto T, Lanaspa MA, Rivard CJ, et al. High-fat and highsucrose (western) diet induces steatohepatitis that is dependent on fructokinase. Hepatology. 2013;58:1632-43.

85. Abdelmalek MF, Suzuki A, Guy C, et al. Increased fructose consumption is associated with fibrosis severity in patients with nonalcoholic fatty liver disease. Hepatology. 2010;51:1961-71.

86. Bugianesi E, Manzini P, D’Antico S, et al. Relative contribution of iron burden, HFE mutations, and insulin resistance to fibrosis in nonalcoholic fatty liver. Hepatology. 2004;39:179-87.

87. Sumida Y, Yoneda M, Hyogo H, et al. A simple clinical scoring system using ferritin, fasting insulin, and type IV collagen $7 \mathrm{~S}$ for predicting steatohepatitis in nonalcoholic fatty liver disease. J Gastroenterol. 2011;46:257-68.

88. Kowdley KV, Belt P, Wilson LA, et al. Serum ferritin is an independent predictor of histologic severity and advanced fibrosis in patients with nonalcoholic fatty liver disease. Hepatology. 2012;55:77-85.

89. Sorrentino P, D'Angelo S, Ferbo U, et al. Liver iron excess in patients with hepatocellular carcinoma developed on non-alcoholic steato-hepatitis. J Hepatol. 2009;50:351-7.

90. Mofrad P, Contos MJ, Haque M, et al. Clinical and histologic spectrum of nonalcoholic fatty liver disease associated with normal ALT values. Hepatology. 2003;37:1286-92.

91. Fracanzani AL, Valenti L, Bugianesi E, et al. Risk of severe liver disease in nonalcoholic fatty liver disease with normal aminotransferase levels: a role for insulin resistance and diabetes. Hepatology. 2008;48:792-8.

92. Yasui K, Hashimoto E, Tokushige K, et al. Clinical and pathological progression of non-alcoholic steatohepatitis to hepatocellular carcinoma. Hepatol Res. 2012;42:767-73.

93. Yoneda M, Fujii H, Sumida Y, et al. Platelet count for predicting fibrosis in nonalcoholic fatty liver disease. J Gastroenterol. 2011;46:1300-6.

94. Wieckowska A, Zein NN, Yerian LM, et al. In vivo assessment of liver cell apoptosis as a novel biomarker of disease severity in nonalcoholic fatty liver disease. Hepatology. 2006;44:27-33.

95. Hui JM, Hodge A, Farrell GC, et al. Beyond insulin resistance in NASH: TNF-alpha or adiponectin? Hepatology. 2004;40:46-54.

96. Yoneda M, Mawatari H, Fujita K, et al. High-sensitivity $\mathrm{C}$-reactive protein is an independent clinical feature of nonalcoholic steatohepatitis (NASH) and also of the severity of fibrosis in NASH. J Gastroenterol. 2007;42:573-82. 
97. Sumida Y, Nakashima T, Yoh T, et al. Serum thioredoxin levels as a predictor of steatohepatitis in patients with nonalcoholic fatty liver disease. J Hepatol. 2003;38:32-8.

98. Takami Y, Uto H, Tamai T, et al. Identification of a novel biomarker for oxidative stress induced by hydrogen peroxide in primary human hepatocytes using the 2-nitrobenzenesulfenyl chloride isotope labeling method. Hepatol Res. 2010;40:438-45.

99. Feldstein AE, Wieckowska A, Lopez AR, et al. Cytokeratin-18 fragment levels as noninvasive biomarkers for nonalcoholic steatohepatitis: a multicenter validation study. Hepatology. 2009;50:1072-8.

100. Kuno A, Ikehara Y, Tanaka Y, et al. A serum "sweet-doughnut" protein facilitates fibrosis evaluation and therapy assessment in patients with viral hepatitis. Sci Rep. 2013;3:1065.

101. Kamada Y, Fujii H, Fujii H, et al. Serum Mac-2 binding protein levels as a novel diagnostic biomarker for prediction of disease severity and nonalcoholic steatohepatitis. Proteomics Clin Appl. 2013;7:648-56.

102. Angulo P, Hui JM, Marchesini G, et al. The NAFLD fibrosis score: a noninvasive system that identifies liver fibrosis in patients with NAFLD. Hepatology. 2007;45:846-54.

103. Shah AG, Lydecker A, Murray K, et al. Comparison of noninvasive markers of fibrosis in patients with nonalcoholic fatty liver disease. Clin Gastroenterol Hepatol. 2009;7:1104-12.

104. Sumida Y, Yoneda M, Hyogo H, et al. Validation of the FIB4 index in a Japanese nonalcoholic fatty liver disease population. BMC Gastroenterol. 2012;12:2.
105. Kim D, Kim WR, Kim HJ, et al. Association between noninvasive fibrosis markers and mortality among adults with nonalcoholic fatty liver disease in the United States. Hepatology. 2013;57:1357-65.

106. Yoneda M, Imajo K, Eguchi $\mathrm{Y}$, et al. Noninvasive scoring systems in patients with nonalcoholic fatty liver disease with normal alanine aminotransferase levels. J Gastroenterol. 2013;48:1051-60.

107. Yoneda M, Yoneda M, Mawatari H, et al. Noninvasive assessment of liver fibrosis by measurement of stiffness in patients with nonalcoholic fatty liver disease (NAFLD). Dig Liver Dis. 2008;40:371-8.

108. Obara N, Ueno Y, Fukushima K, et al. Transient elastography for measurement of liver stiffness measurement can detect early significant hepatic fibrosis in Japanese patients with viral and nonviral liver diseases. J Gastroenterol. 2008;43:720-8.

109. Iijima H, Moriyasu F, Tsuchiya K, et al. Decrease in accumulation of ultrasound contrast microbubbles in non-alcoholic steatohepatitis. Hepatol Res. 2007;37:722-30.

110. Chen J, Talwalkar JA, Yin M, et al. Early detection of nonalcoholic steatohepatitis in patients with nonalcoholic fatty liver disease by using MR elastography. Radiology. 2011;259:749-56.

111. Mittal S, Sada YH, El-Serag HB, et al. Temporal trends of nonalcoholic fatty liver disease-related hepatocellular carcinoma in the veteran affairs population. Clin Gastroenterol Hepatol. 2014. doi:10.1016/j.cgh.2014.08.013. 